\title{
Transient electromagnetic radiation of the lithosphere in a seismically active region
}

\author{
Yiyang Luo ${ }^{1}$, Nguyen Xuan $\mathrm{An}^{2}$, Vladislav Lutsenko ${ }^{3}$, Vladimir Uvarov ${ }^{4}$ \\ ${ }^{1}$ Karazin Kharkiv National University, Ukraine; \\ ${ }^{2}$ Institute of Geophysics, Graduate University of Science and Technology, VAST, Vietnam; \\ ${ }^{3}$ Usikov Institute for Radiophysics and Electronics, NANU, Ukraine; \\ ${ }^{4}$ Institute of Cosmophysical Research and RadioWave propagation FEB RAS, Russia.
}

\begin{abstract}
To study the electromagnetic radiation of the lithosphere associated with seismic waves, we used the recordings of the natural electromagnetic radiation obtained under conditions of weak industrial noise and a high level of microseismicity in the ELF-VLF wave bands. It is shown that these data contain information about the surface waves of the Earth's crust and are accompanied by a frequency close to the first harmonic of the Schumann resonance. The distribution of spikes over thresholds is obtained, which can be indicators of the activity in the processes of the Earth's crust. The averaged form of the spikes for different components of the electromagnetic field is obtained. Attention is drawn to the differences in the various components of the electromagnetic field and their diurnal differences are analyzed. The possibility of using the approach to predict the short-term movement of the Earth's crust is considered.
\end{abstract}

\section{Introduction}

One of the most important problems in modern geophysical earthquake - forecast problems, cannot be considered resolved. To a large extent, this is due to the insufficiency and incompleteness of the information, which was collected by monitoring, lack of knowledge about ongoing processes and, as a consequence of this, with inadequate data processing. There is a special place among the channels of information supply for solving this problem relates to the natural electromagnetic radiation (EMR) of the Earth - extremely informative channel with powerful and constantly operating testing sources covering the entire globe - lightning discharges of the constantly existing thunderstorms. As you know, there are about 3,000 thunderstorms at the same time on Earth, with an average of about 100 lightning strikes per second. In this case, the average dissipated power is about 500 MW, which is enough to power a European city with a population of about 100,000 people. More than $90 \%$ of this energy is converted into EMR, which carries information about the environment of its emergence and propagation. Although research on different aspects of the EMR source from thunderstorms has been conducted for more than a century, there is no comprehensive understanding of the information we got about the nature of the EMR source and the propagation environment.

${ }^{4}$ email for correspondence: uvarovvnng@yandex.ru 
This work deals only with two points of the broad problems, and the solutions of which are necessary for the full interpretation of the information we got:

1. Expanding the frequency range of research on EMR;

2. Analyzing the statistical properties of the recorded EMR.

At present studies of electromagnetic signals related to the lithospheric process in one way or another are one of the least developed problems. To a large extent this is due to insufficient understanding of the interaction process between EMR and the natural propagation medium, which is mainly represented by the lithosphere-atmosphereionosphere system, making it difficult to extract the electromagnetic signals of lithospheric origin from the powerful masking background of thunderstorms, magnetospheric and technical radiations. Under the influence of various factors, there are many mechanisms for forming natural EMR, such as the interaction between lithosphere and ionosphere during earthquakes [1, 2], solar activity, internal gravitational waves (IGW), the atmospheric gravitational waves (AGW) and others [3].

In addition to the above factors for local earthquakes with seismic sources in the oceanic crust, we can consider surface acoustic waves propagating along the free surface of a semi-infinite elastic medium, which can affect surface vibrations of the Earth and acoustic vibrations in the atmosphere [4], which, in turn, can cause ionospheric oscillations.

One of the main approaches is to study the statistical characteristics of EMR which can be used in areas such as earthquake prediction and mineral exploration $[5,6,7]$. The purpose of this research was to study the low-frequency part of the spectra of natural EMR, which may contain information about the electromagnetic manifestation of the crustal surface waves occurring during an earthquake and the changes in the EMR statistical characteristics.

\section{Equipment and methods}

The Statistical characteristics of electromagnetic radiations of lithospheric origin were studied by using registration data of acoustic and electromagnetic radiation ELF-VLF (extremely low and very low frequency) range obtained in the seismically active zone of the Kamchatka [5, 6, 7, 8, 9]. The measurements were carried out on the territory of the expedition center IKIR FEB RAS "Karymshina" located far from populated areas at the intersection of regional fault zones within the Malko-Petropavlovsk zone of the transverse dislocation of the northwestern orientation where the Paratunka spreader zone passes. Magnetic loop antennas with an area of about $10,000 \mathrm{~m} 2$ each located in three mutually perpendicular planes and an electric 8-meter high whip antenna were used [10, 11, 12]. Digital recordings are performed at a sampling frequency of $48 \mathrm{kHz}$ recorded as a 15 minute file and since 2009 regularly conducted.

To analyze the diurnal measurements, the records were divided into frag- ments with a duration of $100 \mathrm{~s}$. After the Fourier transform for each recording segment spectrograms were obtained with a frequency resolution of $0.01 \mathrm{~Hz}$ and a temporal resolution of $100 \mathrm{~s}$.

\section{The aftershock EMR manifestation}

The diurnal series of records of the Earth's natural EMR on December 22 2018, two days after the December 20 earthquake on the Kamchatka Peninsula was investigated (Figure 1). The movement of the Earth's crust in this region was accompanied by active aftershocks but there were no foreshocks. The earthquake and its accompanying aftershocks continued until December 28 and were in the oceanic crust. From December 20 to 28, a total of 58 earthquakes were observed. On December 22 five tremors (aftershocks) 
occurred. Two of which occurred at 13:29:45 and 13:34:20 at noon with maximum intensities with magnitudes of $6 \div 6.5$ points and $5.5 \div 6$ points respectively.

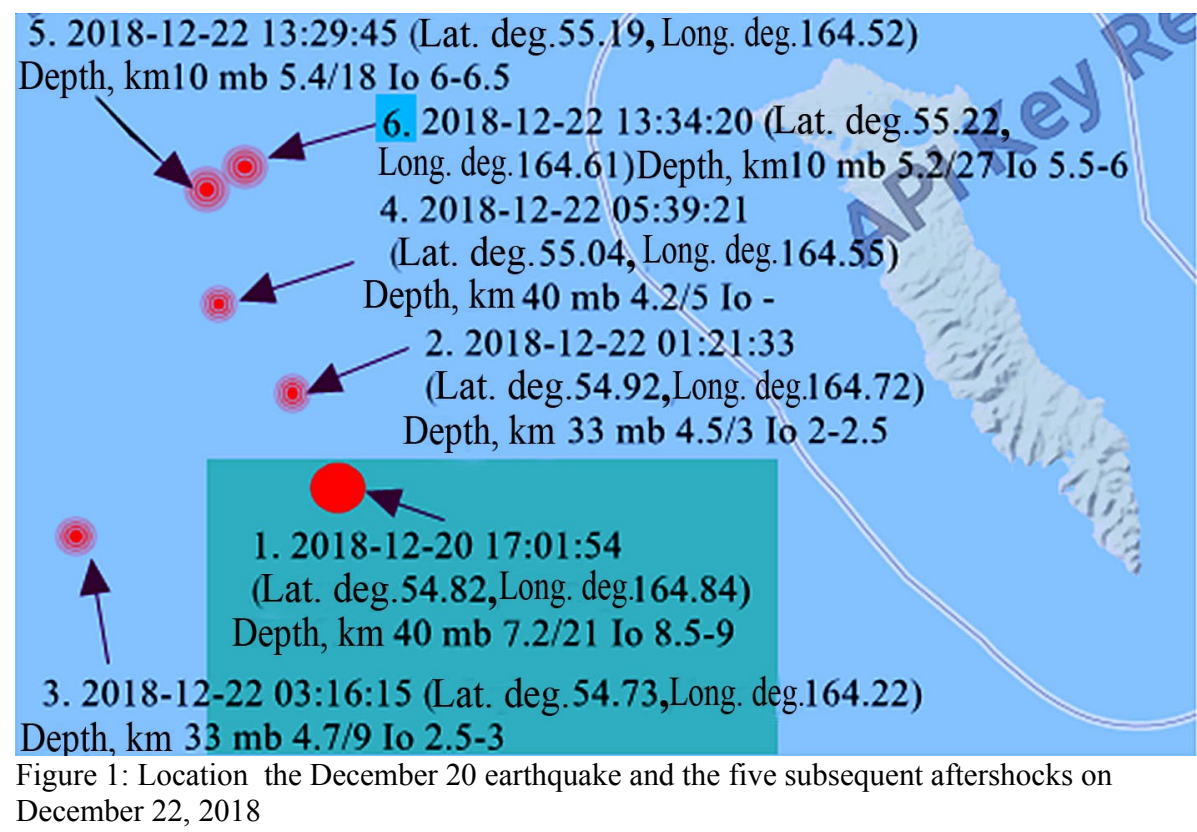

Since according to $[13,14,15]$ the frequency range of Rayleigh waves during earthquakes corresponded to $0 \div 5 \mathrm{~Hz}$. This area served as the object of attention.

The spectrogram (Figure 2) in the frequency range $0 \div 5 \mathrm{~Hz}$ for $\mathrm{Ez}$ and $\mathrm{Hz}$ channels do not contain any significant information. At the same time in this range for $\mathrm{Hy}$ and $\mathrm{Hx}$ channels the difference between daytime and nighttime spectrograms is visible. In addition at some points in time between two tremors and seismic shocks at frequencies of $2 \div 4 \mathrm{~Hz}$, an increased intensity of the spectra is observed.

Given the small depth of the seismic source it can be assumed that the region of increased spectral density in the frequency range of $2 \div 4 \mathrm{~Hz}$ corresponds to the EMR manifestation of the Earth's crustal surface waves, which is consistent with the results of $[13,14,15]$ where surface seismic waves of the same frequency range were detected with small earthquakes. This is the basis to assume that there is an EMR manifestation of Rayleigh waves generated by earthquakes. This effect observed in the horizontal magnetic components can be used in monitoring crustal activity.

\section{Spike over the Thresholds}

The statistical properties of the emissions of the observed parameters in particular, the distribution function of the number of spike over the thresholds depending on the threshold value, reveal the features of the processes and allow us to draw conclusions about their characteristics. The main spike characteristics are the threshold value $\mathrm{T} h$ and average it duration $\tau$.

To make a point belong to a given emission two conditions must be met: 1) the value of the signal s must be higher than the threshold $\mathrm{T} \mathrm{h}: \mathrm{s}>\mathrm{T} \mathrm{h}$; 

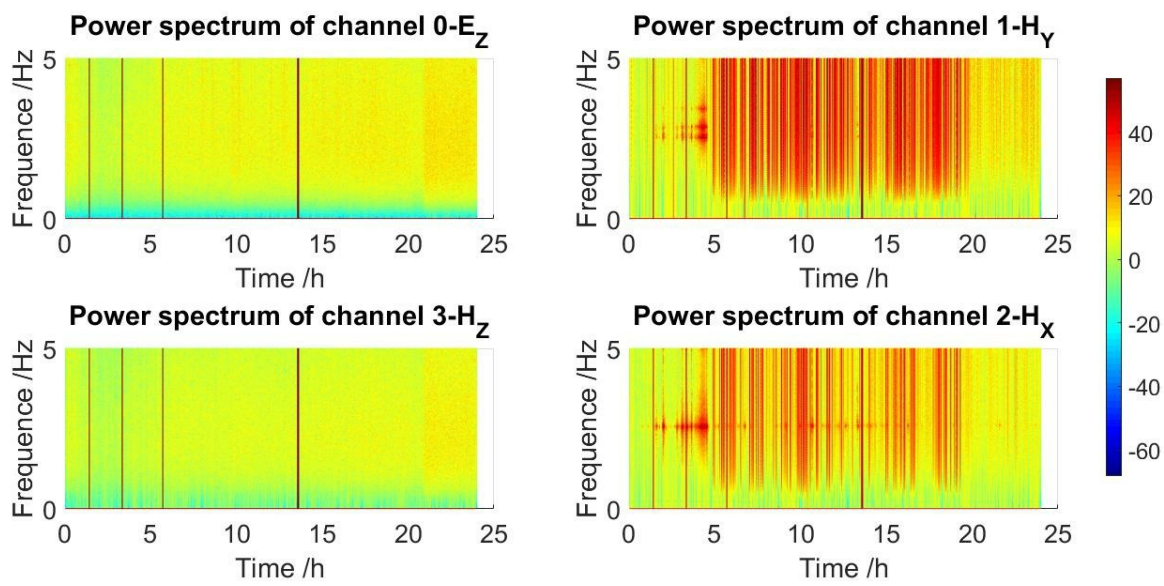

Figure 2: Spectrograms of diurnal measurement in four channels: 0 -Ez; 1 -Hy; 2 -Hx; 3 - Hz. The vertical lines correspond to the moments of aftershocks

2) The time interval $\delta$ t between a given point $i$ and the maximum point of emission imax should not exceed half of the average time duration $\tau: \delta \mathrm{t}=|(\mathrm{ti}-\operatorname{tmax})|<\tau / 2$. Previous studies have shown that the average emission duration usually does not exceed 4 $\mathrm{ms}$ [5].

The Figure 3 shows the curves of the average amplitudes of 15 minute records. It can be seen that the characteristics of the change in the average amplitudes of the respective components of the magnetic field and the electric field during the daytime and nighttime are different.

As can be seen from Figure 3 the minimum value of the amplitude (noise intensity) for the four channels occurs in the 3-channel (vertical magnetic component), which is about 0.0005 . Half of this value is used as the threshold $(\Delta \mathrm{T} h=0,00025)$ and then increase the threshold value $\mathrm{T}=\mathrm{a} \Delta \mathrm{T}$ with an increment of the integer $\mathrm{a}$.
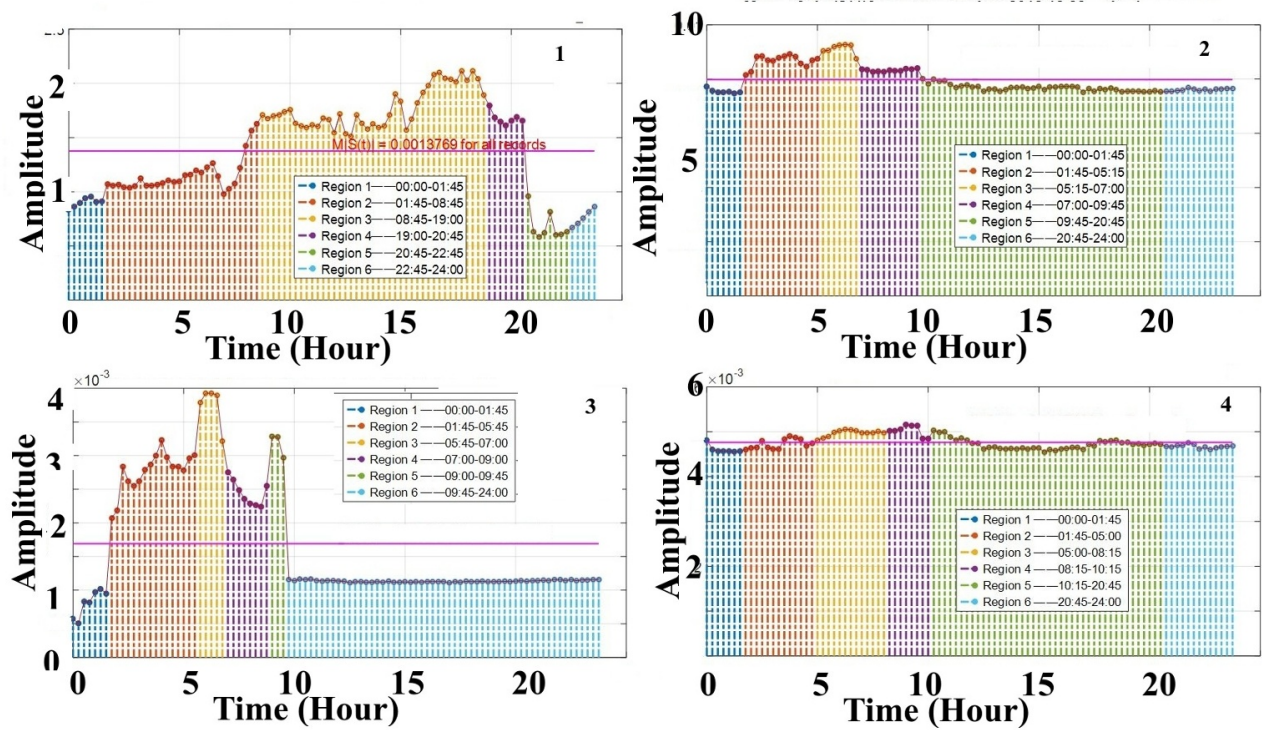

Figure 3: Mean values of the signal amplitudes. 1 - Ez, 2 - Hy, 3 - $\mathrm{Hx}$ and 4 - Hz 
The spikes number exceeding the threshold was calculated. The initial threshold was equal to the diurnal average value of the amplitude. The threshold change step is equal to half of the daily average amplitude.
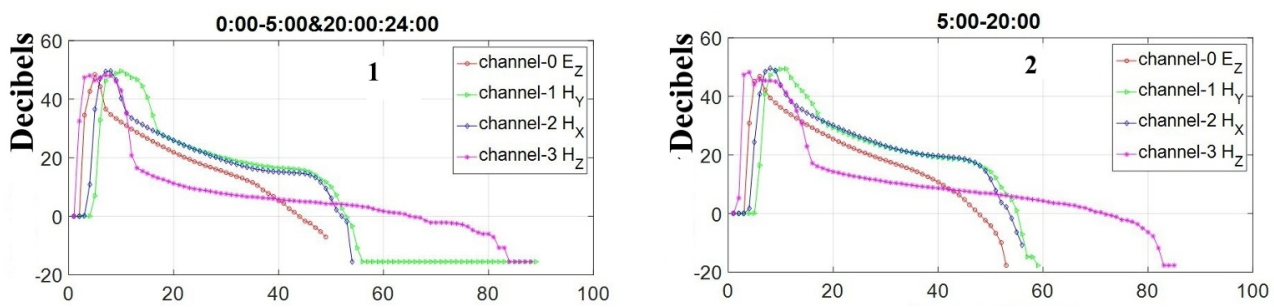

Figure 4: The distribution function of emissions over thresholds: red curves - daytime at local time, green curves - nighttime at local time, in decibels

The distribution of the amount of emissions in each of the channels during the day and night is shown in Figure 4 and the differences in day and night distributions in each channel are shown in Figure 5. Since the durations of the day and night intervals differed significantly, the amount of pulses was normalized by a value proportional to the duration of the observation interval, i.e. the average density of the amount of emissions per observation interval was calculated.

These curves can be divided in to four sections:

1 - section where the emission begins to be detected (interval $1 \div 5$ ), when the extraction algorithm at low thresholds usually cannot attribute continuous pulses to the emission, since they merge with each other;

2 - section of the maximum;

3 - section, which is an almost linearly falling and sufficiently long section, and also the part that we are most interested in the analysis of the process;

4 - section, the final section, characterized by a sharp change in the distribution of the activities of emissions over the threshold which is associated with poor statistical coverage.
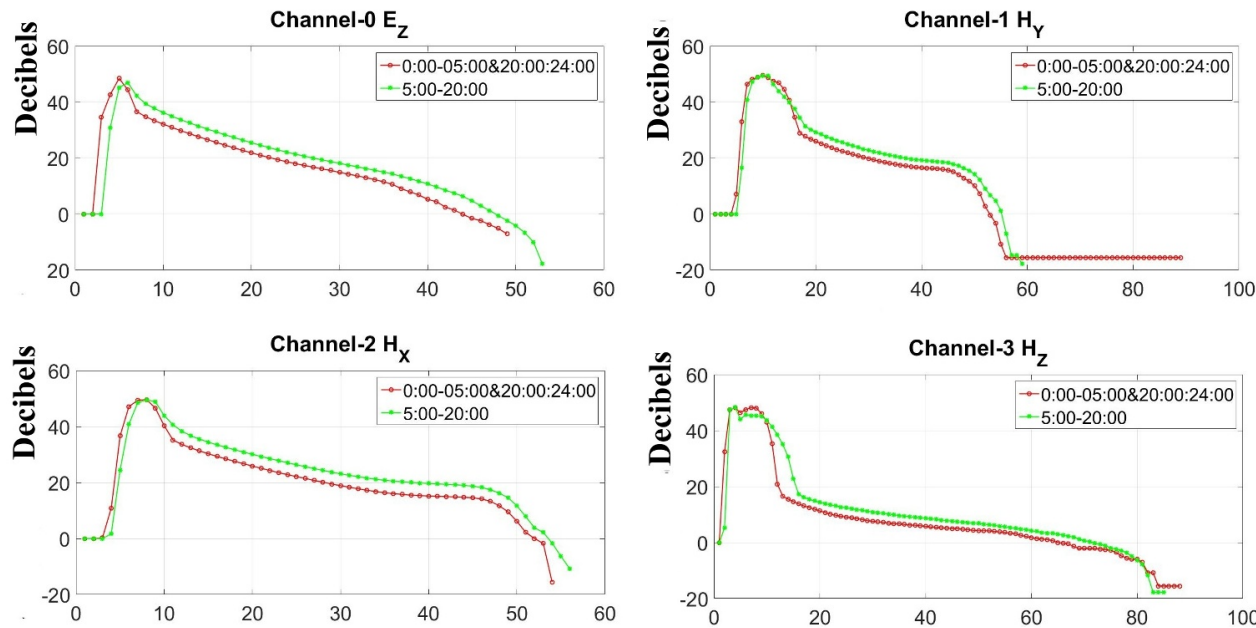

Figure 5: The distribution function of emissions over thresholds: red curves - daytime at local time, green curves - nighttime at local time, in decibels

At any time section $3(20 \div 40)$ is characterized by an almost linear change in the logarithm of the activity of emissions over the threshold, whichsimilar to the dependence of 
the number of earthquakes on its magnitude (Gutenberg-Richter's law). The obtained dependences of the probability of emissions on the threshold are a manifestation of the change in the nature of the processes responsible for their electromagnetic manifestation.

\section{The average waveform of emissions}

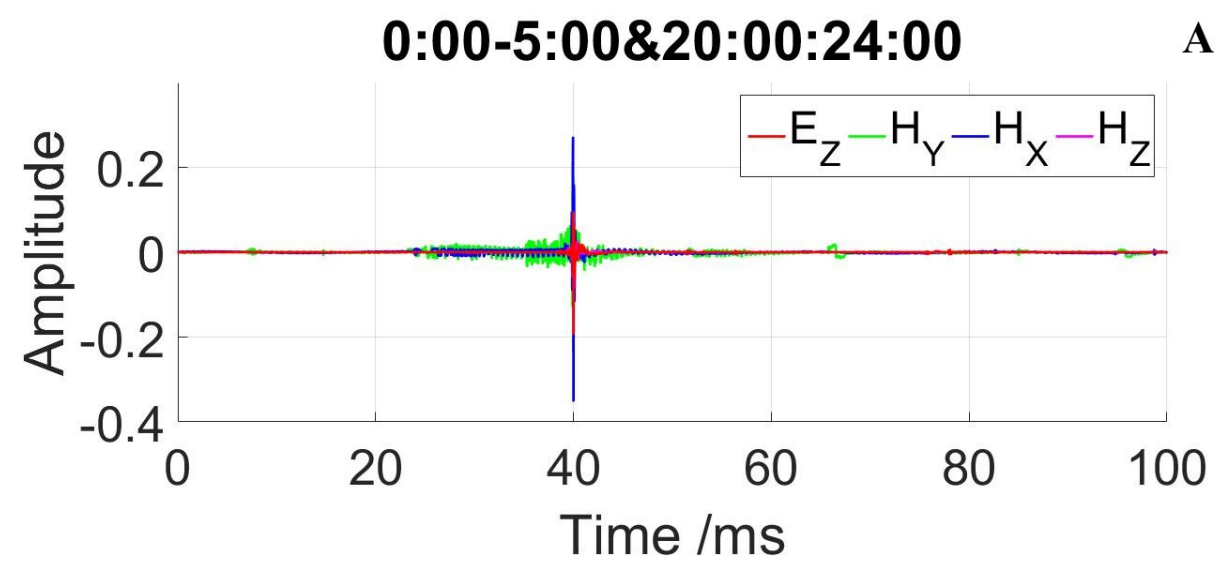

B

\section{5:00-20:00}

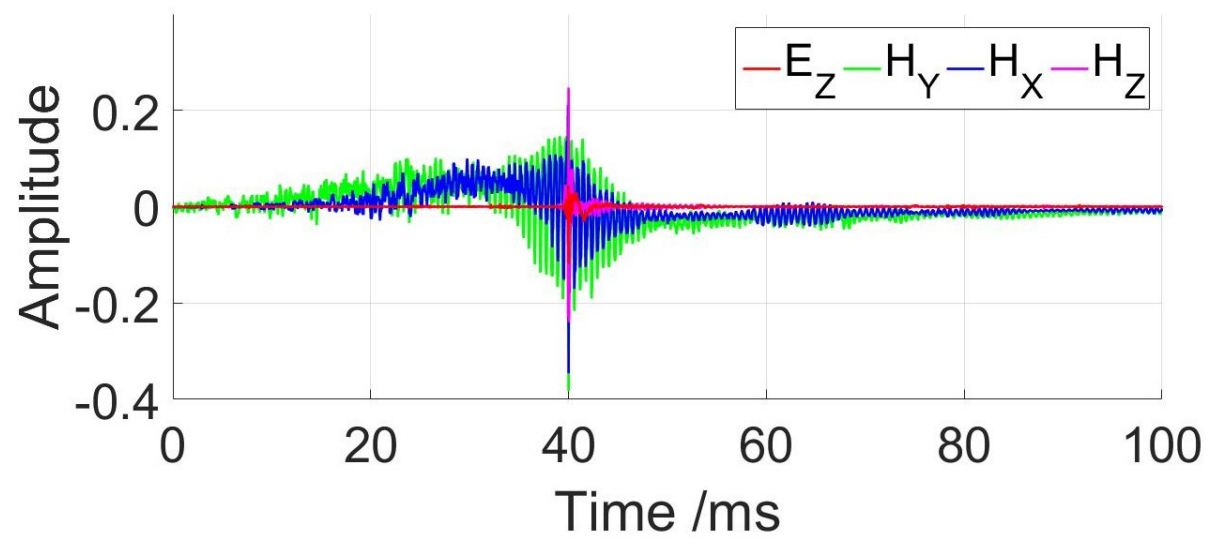

Figure 6: Average waveforms of emissions for different reception channels during the night (A) and day (B) at local time

They may be of interest for monitoring the corresponding processes, including changes in the crustal stresses associated with the preparation of earthquakes. They may be interesting for monitoring the corresponding processes, including changes in crustal stress associated with seismic preparation. The daily change in the distribution of the activity of emissions over the threshold actually reflects the different nature of the daytime and nighttime radiation, probably associated with diurnal variations in the ionosphere.

As can be seen in figure 5 for all channels, after reaching a maximum, the nightly activity is higher than daytime. 

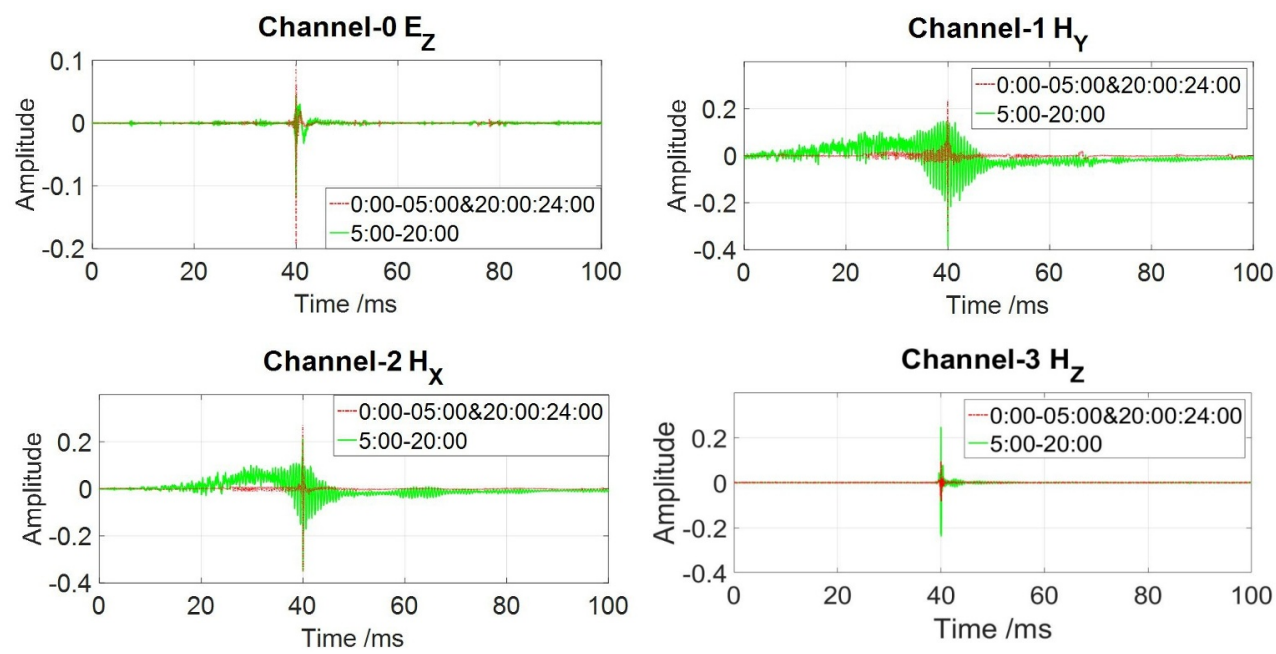

Figure 7: Average waveforms of daytime and nighttime spikes

The average waveform of emissions in the channels was studied, which may reflect the nature of the crustal movements. The waveform of the emissions was determined by the method of accumulation of epochs relative to the moments of maximum values of emissions. In the Fig. 6 shows the obtained average waveforms of emissions during the daytime and nighttime periods. An analysis of the waveforms of the emissions and their spectra shows that the signal of horizontal components of the magnetic field ( $\mathrm{Hy}, \mathrm{Hx}$ ) during the GMT daytime $(5: 00 \div 20: 00$ GMT) have a carrier frequency ofkHz and an envelope with a frequency in the VLF range $(3 \div 30 \mathrm{~Hz})$. Moreover, the frequency with the highest energy is observed near the first-order Schumann resonant frequency. For all components, the waveforms of the emissions initially contain a high-frequency disturbance followed by a low frequency relaxation oscillation, which is the shock excitation characteristic of the resonant system. During the daytime at local time, the duration ofemissions on the horizontal components of the magnetic field is much shorter than in the nighttime. At the same time, for the vertical components of magnetic and electric fields, the difference is much smaller. A comparison of the diurnal changes in the implementation of emissions is shown inFigure 7.

\section{Conclusions}

Electromagnetic emission of the lithosphere contains information that can be used to study geodynamic processes in the cortex. On the one hand, the spectrum of daytime and nighttime emissions of magnetic field components contains manifestations of seismic surface waves that occur within a short time after an earthquake with a frequency in the range of $2 \div 4 \mathrm{~Hz}$. On the other hand, the occurrence of electromagnetic emissions has a diurnal dependence.

The waveform of the average burst at night is accompanied by a frequency close to the first Schumann harmonic $(7.83 \mathrm{~Hz})$.

The distribution of the density of the amount of emissions shows an approximately linear relationship on the thresholds after reaching the maximum. This circumstance can be used to monitor which crust stresses. 
This work was financially supported by RFBR grants 15-05-00543 "Mechanisms of thunderstorm formation under conditions of active volcanic activity on the Kamchatka Peninsula "and with the participation of the Ministry of Industry and Trade of Vietnam.

Graduate student Luo Yiyang thanked for the financial support of the China Scholarship Council (CSC) program ( 201908100008).

\section{References}

[1] M. Gokhberg, S. Shalimov, RJES, 2 (2000)

[2] S. Pulinets, TAO, 15 (2004)

[3] V. Sorokin, M. Hayakawa, MAS, 7 (2013)

[4] S. Watada, T. Kunugi, K. Hirata, H. Kanamori, J. Oikawa, Y. Tsuji, S.

Sekiguchi, GRL, 33 (2006

[5] V. Uvarov, Geofizicheskiy Zhurnal, 34 (2012)

[6] V. Uvarov, Bulletin of KRAESC. Physical - Mathematical Sciences, 14 (2016)

[7] V. Uvarov, G. Druzhin , D. Sannikov, Instruments and Experimental Techniques, 6 (2010)

[8] V. Uvarov, A. Isaev, V. Lutsenko, Proceedings 23th Int. Crimean Conference "Microwave - Telecommunication Technology" (CriMiCo'2013)

[9] V. Kravchenko, O. Kravchenko, V. Uvarov, D. Sannikov, V. Lutsenko, I. Lutsenko, Physical Bases of Instrumentation, 5 (2016)

[10] V. Uvarov, Bulletin of KRAESC. Physical - Mathematical Sciences, 22 (2018)

[11] M. Yasuhiko, O. Yoshihiko, S. Josef, JAE, 27 (2009)

[12] V. Kravchenko, V. Lutsenko, S. Masalov, V. Pustovoit, Doklady Physics, 58 (2013)

[13] A. Kendzera, Geofizicheskiy Zhurnal, 34 (2012)

[14] Yu. Andrushchenko, Geofizicheskiy Zhurnal, 35 (2013)

[15] Yu. Andrushchenko, Geofizicheskiy Zhurnal, 34 (2012) 